\title{
A SEARCH GAME TAKING ACCOUNT OF LINEAR EFFECTS AND LINEAR CONSTRAINTS OF SEARCHING RESOURCE
}

\author{
Ryusuke Hohzaki \\ National Defense Academy
}

(Received March 4, 2011; Revised June 20, 2011)

\begin{abstract}
Search theory has been a major theme in Operations Research. This paper deals with a twoperson zero-sum search game, called search allocation game (SAG), with a searcher and a target as players. In the SAG, the searcher distributes his searching resource in a search space to detect the target while the target moves to evade the searcher. Practical searching resource has a variety of properties on their effects and constraints. A flare fired by a drifting person in the water keeps its brightness within a limited range during some time. Namely, the flare has the property of long-distance effectiveness and temporal durability. There have been so far few researches focusing on the property of searching resource. From a general discussion about the property of searching resource, we notice that linear expressions of variables denoting the resource are crucial to formulate the SAG. In this paper, we propose two linear programming formulations to solve the SAG with linear expressions concerning the effect and the constraints of searching resource and to derive optimal strategies of the searcher and the target.
\end{abstract}

Keywords: Search, search game, game theory, linear programming

\section{Introduction}

Search theory has been a major theme in Operations Research. This paper deals with a two-person zero-sum search game, called search allocation game (SAG), with a searcher and a target as players. In the $\mathrm{SAG}$, the searcher distributes his searching resource in a search space to detect the target while the target moves to evade the searcher. Practical searching resource has a variety of properties on their effects and constraints. A flare fired by a drifting person in the water keeps its brightness within a limited range during some time. Namely, the flare has the property of long-distance effectiveness and temporal durability. There have been so far few researches focusing on the property of searching resource. From a general discussion about the property of searching resource, we notice that linear expressions of variables denoting the resource are crucial to formulate the SAG. In this paper, we propose two linear programming formulations to solve the SAG with linear expressions concerning the effect and the constraints of searching resource and to derive optimal strategies of the searcher and the target.

We are going to survey the past researches on the search problems. Search problems involve two decision makers: a searcher and a searched object or, a 'target'. In the general search, the searcher uses his searching resource to detect the target. Koopman [23] first discussed a search problem to find an optimal distribution of searching resource. Since then, search theory has been a major theme in Operations Research. Koopman's research also motivated the so-called resource allocation problem [16]. de Guenin [8] generalized Koopman's model to an optimal distribution problem of continuous searching resource. Kadane [20] dealt with an optimal distribution of discrete searching resource. In these 
problems, the target was assumed to be stationary. It was natural, in ongoing research, that the stationary target was replaced with a moving target. The first research on the moving target was done by Pollock [26], who discussed an optimal search for a target moving between two cells. Dobbie [5], Hellman [9], Iida [17] and Kan [21] developed similar search models. Stone [27] devoted himself to precise mathematical formulations for a variety of search problems and gathered his results in a book. Brown [1] developed an algorithm to optimize the distribution of continuously divisible searching resource with respect to the detection probability of the target. Washburn [30] refined Brown's method for an optimal distribution of discrete resource. Their research were generalized by Stromquist and Stone [28].

The search problems mentioned above theoretically belong to the convex programming problem, or the global optimization problem, which is concerned with the one-sided decision making of the searcher. In those problems, participants were given the probability law on the distribution of stationary target or the choice of paths by the moving target as prior information. Those one-sided problems progressed to two-sided problems, i.e. search games, with the other decision maker, the target. In almost all search games, the target was assumed to take the moving strategy, as done in one-sided problems. The search game is classified in two ways [7]: search allocation game (SAG), or search-and-evasion game (SAEG), depending on whether the searcher's strategy is the distribution of the searching resource or the moving strategy, respectively. The SAEG model looks easy to understand, and there are many papers, e.g. Danskin [4], Nakai [24] and Kikuta [22], for the model. Washburn [29] discussed a multi-stage game with the traveling time of the searcher as payoff, in which each player decides his next position after being informed of his enemy's current position. Eagle and Washburn [6] dealt with a one-stage game, the payoff of which is the total reward calculated from a sequence of players' positions.

Many efforts also have been expended on the SAG. Nakai [25] and Iida et al. [19] are concerned with SAGs with stationary targets, and Hohzaki and Iida's works $[12,18]$ are the SAG models with moving targets. Especially, Hohzaki and Iida [13] proposed a general method to find an optimal strategy for a convex game of the SAGs. After then, practical conditions or constraints were introduced as assumptions on the target motion, and the SAG models were made more realistic, which brings the models more precision, and more complex solutions. As one example of those complex models, Hohzaki and Washburn [15] introduced energy constraint on the target motion in a SAG defined on a continuous search space. Hohzaki et al. [14] developed a method to solve a SAG with energy constraint in a discrete space, and Hohzaki [10] elucidated a relation between two types of SAGs defined on a continuous space and on a discrete one.

Reviewing past research about the SAG, we notice that almost all researches assume comparatively simple type of searching resource. Only Dambreville and Le Cadre [3] considered several constraints on the amount of resource. They characterize searching resource by some linear constraints. However, in almost all past researches, including Dambreville and Le Cadre's work, constraints are handled just on the amount of resource. Practical searching resource has a variety of properties on its effects and constraints. One of some examples is the flare fired by a drifting person in the water, as I mentioned at the beginning of this section. We can say that the flare is the searching resource with the property of long-distance effectiveness and temporal durability. Many researches, however, neglect these properties of searching resource so far. Only exception is Hohzaki [11], which takes account of concrete attributes of searching resource. When we distill general properties from those practical resources, we notice that linear expressions of variables denoting the searching resource are the key to formulate the SAG with the practical attributes of resource. This 
paper aims to construct a general model for such a SAG and to propose a general method to solve it, considering the linear effects and linear constraints of searching resource.

In the next section, we explain a concrete example of our SAG for comprehensibility, and then describe a general model of the SAG. In Section 3, we derive a linear programming formulation as a methodology for an optimal solution. In Section 4, we point out a shortcoming of the proposed method and generate an additional method to find optimal strategies of the searcher and the target. Using numerical examples, we analyze how sophisticatedly optimal strategies of players are generated in a practical search game on a two-dimensional plane and compare proposed methods for solution by some numerical examples in Section 5.

\section{Modeling and Formulation}

Here we discuss a search game in a general way, where a searcher and a target participate as two players. However we review the mathematical model of Hohzaki [11] to illustrate one example of the search game for comprehensibility before the general discussion. In a search space consisting of time and geographic space, the searcher distributes his searching resource to detect the target, while the target moves to evade the searcher. Effects of searching resource last during some time, and reach a great distance from the resource's dropped point; that is, the searching resource has durability and reachability. Here is a model of this search problem.

(A1) A search space consists of a discrete geographic cell space $\boldsymbol{K}=\{1, \cdots, K\}$ and a discrete time space $\boldsymbol{T}=\{1, \cdots, T\}$. The space is denoted by $\boldsymbol{K} \times \boldsymbol{T}$.

(A2) The target chooses a path running across the search space. A path $\omega$ goes through cell $\omega(t) \in \boldsymbol{K}$ at time $t$. A set of all target paths is denoted by $\Omega$.

(A3) The searcher distributes his searching resource in the search space. His distribution strategy is denoted by $\varphi=\{\varphi(i, t), i \in \boldsymbol{K}, t \in \boldsymbol{T}\}$, where $\varphi(i, t) \in \boldsymbol{R}$ is the nonnegative amount of resource to be distributed in cell $i$ at time $t$. The effectiveness of the resource lasts for time $t_{c}$ after the time it is dropped, and it expands to an area $A(i) \subseteq K$ away from its dropped point $i$, although the intensity of the effectiveness attenuates depending on the distance from the point $i$. The rate of attenuation is denoted by $\beta(i, j)$. As an example of the rate, we might as well assume that $i \in A(i)$ and $\beta(i, i)=1$ for any cell $i$ but $\beta(i, j) \leq 1$ for any other cell $j \in A(i)$ if it is getting smaller at the longer distance.

The searcher can begin to distribute his resource from time $\tau$ and then we denote a time period available for searching by $\widehat{\boldsymbol{T}}=\{\tau, \tau+1, \cdots, T\}$. Two constraints are assumed on the amount of searching resource: upper bound at each time, and the total amount over the entire time, that is,

(a) Upper bound at each time: $\sum_{i \in \boldsymbol{K}} \varphi(i, t) \leq \Phi(t), t \in \widehat{\boldsymbol{T}}$,

(b) Total amount: $\sum_{t=\tau}^{T} \sum_{i \in \boldsymbol{K}} \varphi(i, t) \leq M$.

(A4) For a target path $\omega$ and a distribution plan of searching resource $\varphi$, the searcher can detect the target with probability $1-\exp (-g(\varphi, \omega))$, where $g(\varphi, \omega)$ is a weighted amount of effective resources accumulated over path $\omega$. We weight effective resources accumulated in cell $i$ with $\alpha_{i}$. On detection of the target, the searcher wins reward 1 
but the target loses the same. The searcher gets nothing when he cannot detect the target. The searcher's reward defines the payoff of the game.

We name this model Model A. From Assumption (A3) and (A4), the weighted number $g(\varphi, \omega)$ is calculated as follows. Please note that the detection of the target staying in cell $i=\omega(t)$ at time $t$ is affected by all the resources allocated in an area given by $A^{*}(i) \equiv$ $\{j \mid i \in A(j)\}$ and during a time period from $\max \left\{\tau, t-t_{c}\right\}$ to $t$.

$$
\begin{aligned}
g(\varphi, \omega) & =\sum_{t \in \widehat{\boldsymbol{T}}} \alpha_{\omega(t)} \sum_{\xi=\max \left\{\tau, t-t_{c}\right\}}^{t} \sum_{j \in A^{*}(\omega(t))} \beta(j, \omega(t)) \varphi(j, \xi) \\
& =\sum_{\xi=\tau}^{T} \sum_{t=\xi}^{\min \left\{\xi+t_{c}, T\right\}} \sum_{j \in A^{*}(\omega(t))} \alpha_{\omega(t)} \beta(j, \omega(t)) \varphi(j, \xi) \\
& =\sum_{\xi=\tau}^{T} \sum_{j \in \boldsymbol{K}} \sum_{t=\xi}^{\min \left\{\xi+t_{c}, T\right\}} \delta_{j \in A^{*}(\omega(t))} \alpha_{\omega(t)} \beta(j, \omega(t)) \varphi(j, \xi),
\end{aligned}
$$

where $\delta_{j \in A^{*}(\omega(t))}$ is a kind of the Kronecker's delta defined as 1 if $j \in A^{*}(\omega(t))$ and 0 otherwise. Now we have the payoff of the game, $W(\varphi, \omega)$, from Assumption (A4) and Equation (3).

$$
W(\varphi, \omega)=1-\exp \left(-\sum_{t \in \widehat{T}} \alpha_{\omega(t)} \sum_{\xi=\max \left\{\tau, t-t_{c}\right\}}^{t} \sum_{j \in A^{*}(\omega(t))} \beta(j, \omega(t)) \varphi(j, \xi)\right)
$$

We have so far discussed the search game with durable and reachable searching resource, and we can see that the payoff of the game is given by a function of a linear expression of variable $\varphi(\cdot)$, as in Equation (4). We also must note that constraints on this variable are linear, as seen in Equations (1) and (2). From here we will deal with a generalized search game with some linear expressions of searching resource in its constraints and the payoff of the game, and we will develop a solution method for an equilibrium point. Let us consider a two-person zero-sum game defined in an abstract finite discrete space $\boldsymbol{X}$. We name the following model Model G.

(G1) A target moves in a search space $\boldsymbol{X}$. His pure strategy is to choose one path among a finite set of paths $\Omega$, which is defined as a subset of $\boldsymbol{X}$.

(G2) A searcher distributes his searching resources. We denote his strategy by $\varphi=\{\varphi(x)$, $x \in \boldsymbol{X}\} \in \boldsymbol{R}^{\boldsymbol{X}}$, which is a set of continuous variables defined on $\boldsymbol{X}$. On a strategy $\varphi$, there are $L$ linear constraints $\boldsymbol{a}_{k} \cdot \varphi \leq \Phi_{k}, k \in \boldsymbol{L} \equiv\{1, \cdots, L\}$ and $\varphi \geq \mathbf{0}$, where $\boldsymbol{a}_{k} \cdot \varphi$ is an inner product between two vectors $\boldsymbol{a}_{k}$ and $\varphi$ with dimension $|\boldsymbol{X}|$.

(G3) The payoff is given by $R(\varphi, \omega)=f\left(\boldsymbol{b}_{\omega} \cdot \varphi\right)$ depending on a searcher's strategy $\varphi$ and a target strategy $\omega \in \Omega$. The payoff $R$ represents the total reward the searcher gets in the search operation. The function $f(y)$ is monotone-increasing and concave for $y \in \boldsymbol{R} . \quad \boldsymbol{b}_{\omega} \in \boldsymbol{R}^{\boldsymbol{X}}$ is a parameter defined on $\boldsymbol{X}$ and depends on path $\omega . \boldsymbol{b}_{\omega}(x)$ indicates an effectiveness of resource $\varphi(x)$ to the payoff.

The searcher is a maximizer who desires to maximize the payoff, and the target is a minimizer who wishes to minimize it. 
Here, as a simple example, let us take a basic search game without durable or reachable searching resource, which have been often discussed in Search Theory in the past. The basic assumptions are the same as Assumption (A1) (A4), but they are made by simplifying Assumption (A3) and (A4) like this: No durability and no reachability of searching resource leads us to a simpler function $g(\varphi, \omega)=\sum_{t \in \widehat{T}} \alpha_{\omega(t)} \varphi(\omega(t), t)$ and we assume only upper bound constraint (1) but not (2) on searching resource. This model is an example generated from the general model $(\mathrm{G} 1) \sim(\mathrm{G} 3)$ by setting parameters as follows. Let a search space $\boldsymbol{X}$ be $\boldsymbol{K} \times \boldsymbol{T}$. A target path and a searcher's strategy are defined by $\omega=\{(\omega(t), t) \in \boldsymbol{X}, t \in \boldsymbol{T}\}$ and $\varphi=\{\varphi(i, t),(i, t) \in \boldsymbol{K} \times \widehat{\boldsymbol{T}}\}$. For $\varphi$ and $\omega$, payoff $R(\varphi, \omega)$ is given by an inner product of $\boldsymbol{b}_{\omega}$ and $\varphi, y=\boldsymbol{b}_{\omega} \cdot \varphi$, and function $f(y)=1-\exp (-y)$, where $b_{\omega}(i, t)$ is set as being $\alpha_{i}$ for $i=\omega(t),{ }^{\forall} t \in \widehat{\boldsymbol{T}}$ and 0 otherwise. Feasibility conditions on searching resource are given by $\boldsymbol{a}_{k} \cdot \varphi \leq \Phi_{k}, k \in \widehat{\boldsymbol{T}}$ using $\boldsymbol{L}=\widehat{\boldsymbol{T}}, \boldsymbol{a}_{k}(i, t)=\{1($ for $i \in \boldsymbol{K}, t=k), 0$ (otherwise) $\}, k \in \widehat{\boldsymbol{T}}$ and $\Phi_{k}=\Phi(k), k \in \widehat{\boldsymbol{T}}$.

We name the above model Model Basic. Model A with Assumption (A1) (A4) is also another concrete example of a general model $\mathrm{G}$, of course. Dambreville and Le Cadre [2] illustrate an interesting attribute of searching resource, i.e. renewability. If available resource is renewed up to $\Phi$ every two consecutive time points, we can represent the condition by linear constraints: $\sum_{i \in \boldsymbol{K}}(\varphi(i, t)+\varphi(i, t+1)) \leq \Phi, t=1,3,5, \cdots$.

\section{Equilibrium Point}

Here we develop a method to find an equilibrium point for Model $\mathrm{G}$ with general assumptions $(\mathrm{G} 1) \sim(\mathrm{G} 3)$. We consider a mixed strategy for the target. It can be denoted by $\pi=$ $\{\pi(\omega), \omega \in \Omega\}$, where $\pi(\omega)$ is the probability that the target chooses a path $\omega$. A feasibility region for $\pi$ is

$$
\Pi=\left\{\pi(\omega) \mid \sum_{\omega \in \Omega} \pi(\omega)=1, \pi(\omega) \geq 0, \omega \in \Omega\right\} .
$$

An expected payoff $R(\varphi, \pi)=\sum_{\omega} \pi(\omega) R(\varphi, \omega)$ is linear for $\pi$ and concave for $\varphi$. That is why we are sure that a minimax value of the expected payoff coincides with its maximin value, that is, there exists an equilibrium point, as Hohzaki [13] shows that there is an equilibrium point with a pure strategy of the searcher, $\varphi$, and a mixed strategy of the target, $\pi$, when the payoff is linear for $\pi$ and concave for $\varphi$. From now, we focus on the derivation of an optimal mixed strategy of the target and an optimal pure strategy of the searcher. A feasible region for a searcher's strategy $\varphi$ is given by

$$
\Psi=\left\{\varphi \mid \boldsymbol{a}_{k} \cdot \varphi \leq \Phi_{k}, k \in \boldsymbol{L}, \varphi(x) \geq 0, x \in \boldsymbol{X}\right\} .
$$

Taking account of the region (5) and the monotonic increasingness of the function $f(\cdot)$, we can transform the maximin optimization problem as follows.

$$
\max _{\varphi \in \Psi} \min _{\pi \in \Pi} R(\varphi, \pi)=\max _{\varphi \in \Psi} \min _{\omega \in \Omega} R(\varphi, \omega)=\max _{\varphi \in \Psi} \min _{\omega \in \Omega} f\left(\boldsymbol{b}_{\omega} \cdot \varphi\right)=f\left(\max _{\varphi \in \Psi} \min _{\omega \in \Omega} \boldsymbol{b}_{\omega} \cdot \varphi\right) .
$$

An optimization problem lying in parentheses in the last expression is solved by the following linear programming problem.

$$
\begin{array}{ll}
G^{S}: & \max _{\varphi} \eta \\
\text { s.t. } & \boldsymbol{b}_{\omega} \cdot \varphi \geq \eta, \omega \in \Omega \\
& \boldsymbol{a}_{k} \cdot \varphi \leq \Phi_{k}, \quad k \in \boldsymbol{L} \\
& \varphi(x) \geq 0, \quad x \in \boldsymbol{X} .
\end{array}
$$


From the above formulation, we can find an optimal strategy of the searcher $\varphi^{*}=\left\{\varphi^{*}(x), x \in\right.$ $\boldsymbol{X}\}$ and calculate a maximin value of $R(\varphi, \pi)$ by $f\left(\eta^{*}\right)$ using an optimal value of $\left(G^{S}\right), \eta^{*}$. We have to note that problem $\left(G^{S}\right)$ is nothing but a maximin problem for a redefined expected payoff $R(\varphi, \pi)=\sum_{\omega} \pi(\omega) \boldsymbol{b}_{\omega} \cdot \varphi$. That is why from now we take this bilinear expression of variables $\pi$ and $\varphi$ as the expected payoff.

If we set dual variables $\mu(\omega)$ and $\nu_{k}$ corresponding to conditions (7) and (8), respectively, we have the following dual problem to $\left(G^{S}\right)$.

$$
\begin{array}{ll}
D^{S}: & \min _{\mu(\omega), \nu_{k}} \sum_{k \in \boldsymbol{L}} \Phi_{k} \nu_{k} \\
\text { s.t. } & \sum_{\omega \in \Omega} \mu(\omega)=1 \\
& \mu(\omega) \geq 0, \omega \in \Omega \\
& -\sum_{\omega \in \Omega} b_{\omega}(x) \mu(\omega)+\sum_{k \in \boldsymbol{L}} a_{k}(x) \nu_{k} \geq 0, x \in \boldsymbol{X} \\
& \nu_{k} \geq 0, \quad k \in \boldsymbol{L} .
\end{array}
$$

We notice that conditions (9) and (10) are the same as those in Equation (5). Really we can prove that optimal $\mu(\omega)$ gives an optimal mixed strategy of the target $\pi^{*}(\omega)$ as follows.

A maximization problem of the expected payoff is

$$
\max _{\varphi} \sum_{\omega \in \Omega} \pi(\omega) \boldsymbol{b}_{\omega} \cdot \varphi \quad \text { s.t. } \quad \boldsymbol{a}_{k} \cdot \varphi \leq \Phi_{k}, k \in \boldsymbol{L}, \varphi(x) \geq 0, x \in \boldsymbol{X} .
$$

As a dual problem to the above, we have the following formulation, the optimal value of which is the same as that of the primal problem.

$$
D^{T}: \min _{\epsilon_{k}} \sum_{k \in \boldsymbol{L}} \Phi_{k} \epsilon_{k} \quad \text { s.t. } \quad \sum_{k \in \boldsymbol{L}} a_{k}(x) \epsilon_{k} \geq \sum_{\omega \in \Omega} \pi(\omega) b_{\omega}(x), x \in \boldsymbol{X}, \quad \epsilon_{k} \geq 0, k \in \boldsymbol{L} .
$$

To find a minimax value of the expected payoff, let us minimize further the value of problem $\left(D^{T}\right)$ with respect to $\pi(\omega)$ to get

$$
\begin{array}{ll}
G^{T}: & \min _{\pi(\omega), \epsilon_{k}} \sum_{k \in \boldsymbol{L}} \Phi_{k} \epsilon_{k} \\
\text { s.t. } & \sum_{k \in \boldsymbol{L}} a_{k}(x) \epsilon_{k}-\sum_{\omega \in \Omega} \pi(\omega) b_{\omega}(x) \geq 0, x \in \boldsymbol{X} \\
& \epsilon_{k} \geq 0, k \in \boldsymbol{L} \\
& \sum_{\omega \in \Omega} \pi(\omega)=1 \\
& \pi(\omega) \geq 0, \omega \in \Omega .
\end{array}
$$

After comparing problems $\left(G^{T}\right)$ and $\left(D^{S}\right)$, we can see that $\pi(\omega)$ and $\epsilon_{k}$ are equivalent to $\mu(\omega)$ and $\nu_{k}$, respectively. We can state our results as a theorem.

Theorem 1. An optimal strategy of the searcher $\varphi^{*}(x)$ is given as a solution of Problem $\left(G^{S}\right)$ and an optimal strategy of the target $\pi^{*}(\omega)$ is given as an optimal dual variable corresponding to condition (7). On the other hand, Problem $\left(G^{T}\right)$ gives us an optimal target 
strategy $\pi^{*}(\omega)$ as its optimal solution and an optimal dual variable to condition (11) brings us an optimal searcher strategy $\varphi^{*}(x)$. $\left(G^{T}\right)$.

We can calculate the value of the game by $f\left(\eta^{*}\right)$ using an optimal value $\eta^{*}$ of $\left(G^{S}\right)$ or

In Table 1, we list the parameter setting of Model G when we apply the model to Model Basic and Model A. In the last row of the table, we use notation

$$
\gamma_{i t}(\pi) \equiv \sum_{\xi=t}^{\min \left\{t+t_{c}, T\right\}} \sum_{j \in A(i)}\left(\sum_{\omega \in \Omega_{j \xi}} \pi(\omega)\right) \alpha_{j} \beta(i, j)
$$

and $\Omega_{j \xi} \equiv\{\omega \in \Omega \mid \omega(\xi)=j\}$ which indicates a set of target paths running through cell $j$ at time $\xi$.

Table 1: Parameter setting for Model Basic and Model A

\begin{tabular}{|c|c|c|}
\hline Model $G$ & Model Basic & Model $A$ \\
\hline$L$ & $\widehat{T}$ & $\widehat{\boldsymbol{T}} \cup\{T+1\}$ \\
\hline$X$ & $\bar{K} \times T$ & $K \times T$ \\
\hline$x \in X$ & $(i, t) \in \boldsymbol{K} \times \boldsymbol{T}$ & $(i, t) \in \boldsymbol{K} \times \boldsymbol{T}$ \\
\hline$a_{k}(x)$ & $\begin{array}{l}\text { for }{ }^{\forall} t \in \widehat{\boldsymbol{T}}, \\
a_{k}(i, t)=\left\{\begin{array}{l}1(\text { for } i \in \boldsymbol{K}, k=t) \\
0(\text { othewise })\end{array}\right.\end{array}$ & $\begin{array}{l}\text { for }{ }^{\forall} t \in \widehat{\boldsymbol{T}}, \\
a_{k}(i, t)=\left\{\begin{array}{l}1(\text { for } i \in \boldsymbol{K}, k=t) \\
0(\text { othewise })\end{array}\right. \\
a_{T+1}(i, t)=1,{ }^{\forall}(i, t) \in \boldsymbol{K} \times \widehat{\boldsymbol{T}}\end{array}$ \\
\hline$b_{\omega}(x)$ & $\begin{array}{l}\text { for }{ }^{\forall} t \in \widehat{\boldsymbol{T}} \\
b_{\omega}(i, t)=\left\{\begin{array}{l}\alpha_{i}(\text { for } i=\omega(t)) \\
0(\text { otherwise })\end{array}\right.\end{array}$ & $\begin{array}{l}\text { for }{ }^{\forall} t \in \widehat{\boldsymbol{T}}, \quad b_{\omega}(i, t) \\
=\sum_{\xi=t}^{\min \left\{t+t_{c}, T\right\}} \delta_{i \in A^{*}(\omega(\xi))} \alpha_{\omega(\xi)} \beta(i, \omega(\xi))\end{array}$ \\
\hline$\Phi_{k}, k \in \boldsymbol{L}$ & $\Phi_{k}=\Phi(k), k \in \widehat{T}$ & $\Phi_{k}=\Phi(k), k \in \widehat{\boldsymbol{T}}, \Phi_{T+1}=M$ \\
\hline $\begin{array}{c}\sum_{k \in \boldsymbol{L}} a_{k}(x) \epsilon_{k} \\
-\sum_{\omega \in \Omega} \pi(\omega) b_{\omega}(x) \geq 0\end{array}$ & $\begin{array}{l}\epsilon_{t}-\sum_{\omega \in \Omega_{i t}} \pi(\omega) \alpha_{i} \geq 0 \\
\quad \text { for } x=(i, t) \in \boldsymbol{K} \times \widehat{\boldsymbol{T}}\end{array}$ & $\begin{array}{l}\epsilon_{t}+\epsilon_{T+1}-\gamma_{i t}(\pi) \geq 0 \\
\quad \text { for } x=(i, t) \in \boldsymbol{K} \times \widehat{\boldsymbol{T}}\end{array}$ \\
\hline
\end{tabular}

Using expressions in the last row, let us write down Problem $\left(G^{T}\right)$ for Model Basic. Condition (12) is not necessarily required because $\epsilon_{k}$ becomes nonnegative from other conditions.

$$
\begin{array}{ll}
P_{B}^{T} \quad: \quad \min _{\pi, \epsilon} \sum_{\xi=\tau}^{T} \Phi(\xi) \epsilon_{\xi} \\
\text { s.t. } \quad \alpha_{i} \sum_{\omega \in \Omega_{i t}} \pi(\omega) \leq \epsilon_{t}, \quad i \in \boldsymbol{K}, t \in \widehat{\boldsymbol{T}}, \quad \sum_{\omega \in \Omega} \pi(\omega)=1, \quad \pi(\omega) \geq 0, \omega \in \Omega .
\end{array}
$$

Applying Table 1 to Model A, we obtain the following problem.

$$
\begin{array}{ll}
P_{A}^{T}: & \min _{\pi, \epsilon} \sum_{\xi \in \widehat{\boldsymbol{T}}} \Phi(\xi) \epsilon_{\xi}+M \epsilon_{T+1} \\
\text { s.t. } & \sum_{\xi=t}^{\min \left\{t+t_{c}, T\right\}} \sum_{j \in A(i)}\left(\sum_{\omega \in \Omega_{j \xi}} \pi(\omega)\right) \alpha_{j} \beta(i, j) \leq \epsilon_{t}+\epsilon_{T+1}, i \in \boldsymbol{K}, t \in \widehat{\boldsymbol{T}} \\
& \epsilon_{t} \geq 0, t \in \widehat{\boldsymbol{T}} \cup\{T+1\}, \quad \sum_{\omega \in \Omega} \pi(\omega)=1, \quad \pi(\omega) \geq 0, \omega \in \Omega .
\end{array}
$$




\section{Another Formulation Explicitly Involving Path Constraints}

In Assumption (G1) of Model G and Assumption (A2) of Model A, we assume that the target paths $\Omega$ are given in advance and don't give any constraint on the target path, explicitly. No constraint gives Problem $\left(G^{S}\right)$ and $\left(G^{T}\right)$ flexibilities to make these problems suitable to some special cases of feasible paths by adding concrete path constraints and materializing $\Omega$ if necessary. However in terms of the size of $\Omega$, we cannot say that these problem formulations are practical or feasible for the large size of the search space $\boldsymbol{X}$ or $\boldsymbol{K} \times \boldsymbol{T}$. For example, if there is no constraint on $\Omega$ in the finite search space $\boldsymbol{K} \times \boldsymbol{T}$ of Assumption (A1), we can count the number of paths up to $\left|\boldsymbol{K} \|^{\boldsymbol{T}}\right|$ and the number would be too huge to solve even the linear programming problem such as $\left(G^{S}\right)$ or $\left(G^{T}\right)$. To cope with this computational difficulty, in this section, we adopt another type of target strategy, say existence probability and transition probability, and discuss another formulation without enumerating target paths.

First of all, let us begin with assuming some concrete conditions on the target path in a search space $\boldsymbol{X}=\boldsymbol{K} \times \boldsymbol{T}$ of a geographic space $\boldsymbol{K}$ and time space $\boldsymbol{T}$. For the target, we provide a set of attributes to represent his state. We itemize the full specification of a new model with a linear form of payoff, as follows. The linear payoff is essentially equivalent to a concave and monotone increasing payoff, as stated before.

(C1) A search space consists of a finite discrete time space $\boldsymbol{T}=\{1, \cdots, T\}$ and a finite discrete geographic space $\boldsymbol{K}_{t}$, which varies depending on time $t \in \boldsymbol{T}$.

(C2) A target strategy is to choose one path among a set of feasible paths, $\Omega$, running through the space. On a path $\omega \in \Omega$, he is in cell $\omega(t) \in \boldsymbol{K}_{t}$ at time $t$ and at the same time he must be in a state $s$ among a set $\boldsymbol{S}_{t}$. From a state $(i, t, s)$, which represents being in cell $i$ at time $t$ with attribute $s$, the target can move to only a set of cells $B(i, t, s)$ at the next time $t+1$. If he moves to cell $j \in B(i, t, s)$, his current attribute $s$ changes to an attribute $\Gamma(i, t, s, j)$ at time $t+1$. Equation $u=\Gamma(i, t, s, j)$ is assumed to have a one-to-one correspondence between $u$ and $s$ for any $i, t$ and $j$.

(C3) A searcher strategy is to distribute his searching resource in the search space. A strategy is denoted by $\varphi=\left\{\varphi(i, t), i \in \boldsymbol{K}_{t}, t \in \boldsymbol{T}\right\}$, where $\varphi(i, t)$ is the amount of resource to be allocated in cell $i$ at time $t$. On $\varphi$, there are $L$ linear constraints $\boldsymbol{a}_{l} \cdot \varphi \leq \Phi_{l}, l \in \boldsymbol{L} \equiv\{1, \cdots, L\}$ and $\varphi \geq \mathbf{0}$, where $\boldsymbol{a}_{l} \cdot \varphi$ is an inner product $\sum_{t \in \boldsymbol{T}} \sum_{i \in \boldsymbol{K}_{t}} \boldsymbol{a}_{l}(i, t) \varphi(i, t)$.

(C4) A searcher strategy $\varphi$ and a target strategy $\omega$ yield a payoff $R(\varphi, \omega)=\boldsymbol{b}_{\omega} \cdot \varphi$. An element $\boldsymbol{b}_{\omega}(i, t)$ of a vector $\boldsymbol{b}_{\omega}$ is determined by a sum of values $c(i, \omega(\xi), \xi)$ with respect to $\omega(\xi)$ over an area of cells $A(i, \xi)$ while time index $\xi$ varies through a time period $T(t) \subseteq \boldsymbol{T}$ with time base $t$, that is,

$$
\boldsymbol{b}_{\omega}(i, t)=\sum_{\xi \in T(t)} \delta_{\omega(\xi) \in A(i, \xi)} c(i, \omega(\xi), \xi)
$$

where $\delta_{\omega(\xi) \in A(i, \xi)}$ is 1 if $\omega(\xi) \in A(i, \xi)$ and 0 otherwise. Time period $T(t)$ is assumed to contain its time base $t$, that is, $t \in T(t)$. Area $A(i, \xi)$ contains its cell base $i$, $i \in A(i, \xi)$, and it is set as a subset of $\boldsymbol{K}_{\xi}$, that is, $A(i, \xi) \subseteq \boldsymbol{K}_{\xi}$.

The searcher is a maximizer who wants to maximize the payoff and the target is a minimizer with his desire to minimize it.

We can think of the moving energy of the target as an example of the attribute $S_{t}$ in (C2). The possession of much energy would give the target high mobility and his residual 
energy would be getting smaller while moving. Coefficient $c(i, \omega(\xi), \xi)$ in (C4) represents an element of influence by searching resource on the payoff $R(\varphi, \omega)$, as we can see from Equation (3) in terms of the long-distance effectiveness and temporal durability of resource.

We already have developed a formulation for the value of the game in Section 3. We can begin our discussion from the formulation $\left(G^{T}\right)$. We are going to redefine a strategy of the target and rewrite the formulation $\left(G^{T}\right)$ by the newly-defined strategy. We can expand the second term in condition (11) as follows.

$$
\begin{aligned}
& \sum_{\omega \in \Omega} \pi(\omega) b_{\omega}(i, t)=\sum_{\omega \in \Omega} \pi(\omega) \sum_{\xi \in T(t)} \delta_{\omega(\xi) \in A(i, \xi)} c(i, \omega(\xi), \xi) \\
& =\sum_{\omega \in \Omega} \pi(\omega) \sum_{\xi \in T(t)} \sum_{k \in A(i, \xi)} \delta_{k \omega(\xi)} c(i, k, \xi)=\sum_{\xi \in T(t)} \sum_{k \in A(i, \xi)}\left(\sum_{\omega \in \Omega} \pi(\omega) \delta_{k \omega(\xi)}\right) c(i, k, \xi)
\end{aligned}
$$

In the above, $\sum_{\omega \in \Omega} \pi(\omega) \delta_{k \omega(\xi)}$ is a sum of target probabilities gathering in cell $k$ at time $\xi$. This is one of reasons that we adopt the following variables as a target strategy instead of choosing paths.

We represent a state of the target by a triplet $(i, t, s)$, which indicates that the target is in cell $i$ at time $t$ with attribute $s$. Let $q(i, t, s)$ be the probability that the target is in state $(i, t, s)$ and $v(i, j, t, s)$ be the probability that the target is in state $(i, t, s)$ and it moves to cell $j$ at next time $t+1$. Using these new variables, we can replace $\sum_{\omega \in \Omega} \pi(\omega) \delta_{k \omega(\xi)}$ with $\sum_{s \in \boldsymbol{S}_{\xi}} q(k, \xi, s)$. By variables $q(\cdot)$ and $v(\cdot)$, feasibility conditions (13) and (14) can be rewritten in some expressions, which is referred to as so-called the conservation law of probability flows.

$$
\begin{array}{ll}
C^{T}: & \min _{q, v, \epsilon_{k}} \sum_{l \in \boldsymbol{L}} \Phi_{l} \epsilon_{l} \\
\text { s.t. } \quad & \sum_{l \in \boldsymbol{L}} a_{l}(i, t) \epsilon_{k}-\sum_{\xi \in T(t)} \sum_{k \in A(i, \xi)} \sum_{s \in \boldsymbol{S}_{\xi}} q(k, \xi, s) c(i, k, \xi) \geq 0, i \in \boldsymbol{K}_{t}, t \in \boldsymbol{T} \\
& \epsilon_{l} \geq 0, l \in \boldsymbol{L} \\
& q(i, t, s)=\sum_{j \in B(i, t, s)} v(i, j, t, s), i \in \boldsymbol{K}_{t}, s \in \boldsymbol{S}_{t}, t=1, \cdots, T-1 \\
& q(i, t, s)=\sum_{j \in B^{*}(i, t, s)} v\left(j, i, t-1, \Gamma^{*}(j, i, t, s)\right), i \in \boldsymbol{K}_{t}, s \in \boldsymbol{S}_{t}, t=2, \cdots, T \\
& \sum_{i \in \boldsymbol{K}_{t}} \sum_{s \in \boldsymbol{S}_{t}}{ }_{q(i, t, s)=1, t \in \boldsymbol{T}} \\
& v(i, j, t, e) \geq 0, i \in \boldsymbol{K}_{t}, s \in \boldsymbol{S}_{t}, j \in B(i, t, s), t=1, \cdots, T-1
\end{array}
$$

$\Gamma^{*}(j, i, t, s)$ is an inverse function obtained by solving an equation $s=\Gamma(j, t-1, u, i)$ with respect to $u$. It indicates a target attribute at time $t-1$, with which the target is transferable to state $(i, t, s)$ from cell $j . B^{*}(i, t, s)$ indicates a set of cells, from which the target can move to state $(i, t, s)$ and it is an inverse function of $B(\cdot)$, defined by

$$
B^{*}(i, t, s) \equiv\left\{j \in \boldsymbol{K}_{t-1} \mid i \in B\left(j, t-1, \Gamma^{*}(j, i, t, s)\right)\right\}
$$

Equation (18) is set so as to conserve out-flow probabilities of the target from state $(i, t, s)$ and Equation (19) is for in-flow probabilities into $(i, t, s)$. Equation (20) secures that the 
target should be always somewhere in a geographic area $\boldsymbol{K}_{t}$ with one of attributes $\boldsymbol{S}_{t}$. The transition probability $v(\cdot)$ must be nonnegative as shown in condition (21).

Problem $\left(C^{T}\right)$ gives us the value of the game and an optimal target strategy as its existence probability and transition probability. Next we turn our attention to a procedure to find an optimal strategy of the searcher. It seems difficult to replace original target path $\omega$ with representative variables $q(\cdot), v(\cdot)$ in Problem $\left(G^{S}\right)$. So we give up using the formulation $\left(G^{S}\right)$ directly and try another approach. From Assumption (C4) and the transformation (16), we can rewrite the expected payoff $\sum_{\omega \in \Omega} \pi(\omega) \boldsymbol{b}_{\omega} \cdot \varphi$ by variable $q(\cdot)$, as follows.

$$
\begin{aligned}
\sum_{\omega \in \Omega} \pi(\omega) \boldsymbol{b}_{\omega} \cdot \varphi & =\sum_{t \in \boldsymbol{T}} \sum_{i \in \boldsymbol{K}_{t}}\left(\sum_{\xi \in T(t)} \sum_{k \in A(i, \xi)} \sum_{s \in \boldsymbol{S}_{\xi}} q(k, \xi, s) c(i, k, \xi)\right) \varphi(i, t) \\
& =\sum_{\xi \in \boldsymbol{T}} \sum_{k \in \boldsymbol{K}_{\xi}} \sum_{s \in \boldsymbol{S}_{\xi}}\left(\sum_{t \in T^{*}(\xi)} \sum_{i \in A^{*}(k, \xi)} c(i, k, \xi) \varphi(i, t)\right) q(k, \xi, s),
\end{aligned}
$$

where $T^{*}(\xi) \equiv\{t \in \boldsymbol{T} \mid \xi \in T(t)\}$ and $A^{*}(k, \xi) \equiv\left\{i \in \boldsymbol{K}_{\xi} \mid k \in A(i, \xi)\right\}$.

We define a new value $w(k, \xi, s)$ as the minimum payoff yielded by an optimal target path after time $\xi$ when the target starts from state $(k, \xi, s)$. Now that the target is in state $(k, \xi, s)$ and then $q(k, \xi, s)=1$, there occurs payoff $\sum_{t \in T^{*}(\xi)} \sum_{i \in A^{*}(k, \xi)} c(i, k, \xi) \varphi(i, t)$ at the time $\xi$, as seen from Equation (22). If the target moves to cell $j$ at next time $\xi+1$, the minimum payoff after the time is given by $w(j, \xi+1, \Gamma(k, \xi, s, j))$. Now we have the following dynamic programming formulation with regard to value $w(k, \xi, s)$.

$$
w(k, \xi, s)=\min _{j \in B(k, \xi, s)}\left\{\sum_{t \in T^{*}(\xi)} \sum_{i \in A^{*}(k, \xi)} c(i, k, \xi) \varphi(i, t)+w(j, \xi+1, \Gamma(k, \xi, s, j))\right\}
$$

An initial value of $w(k, \xi, s)$ is definitely determined by

$$
w(k, T, s)=\sum_{t \in T^{*}(T)} \sum_{i \in A^{*}(k, T)} c(i, k, T) \varphi(i, t)
$$

at the last time $t=T$.

Because the minimum payoff over the whole search is $\min _{k \in \boldsymbol{K}_{1}, s \in \boldsymbol{S}_{1}} w(k, 1, s)$, the searcher distributes his searching resource so as to maximize the payoff. In consequence, an optimal searcher strategy $\varphi^{*}$ is given by a solution of the following linear programming problem. The problem also bears a maximin value of the expected payoff.

$$
\begin{array}{ll}
C^{S}: & \max _{\varphi, \rho} \rho \\
\text { s.t. } & \rho \leq w(k, 1, s), k \in \boldsymbol{K}_{1}, s \in \boldsymbol{S}_{1} \\
& w(k, \xi, s) \leq \sum_{t \in T^{*}(\xi)} \sum_{i \in A^{*}(k, \xi)} c(i, k, \xi) \varphi(i, t)+w(j, \xi+1, \Gamma(k, \xi, s, j)), \\
& \quad k \in \boldsymbol{K}_{\xi}, j \in B(k, \xi, s), s \in \boldsymbol{S}_{\xi}, \xi=1, \cdots, T-1 \\
& w(k, T, s)=\sum_{t \in T^{*}(T)} \sum_{i \in A^{*}(k, T)} c(i, k, T) \varphi(i, t), k \in \boldsymbol{K}_{T}, s \in \boldsymbol{S}_{T} \\
& \sum_{t \in \boldsymbol{T}} \sum_{i \in \boldsymbol{K}_{t}} \boldsymbol{a}_{l}(i, t) \varphi(i, t) \leq \Phi_{l}, l \in \boldsymbol{L}, \varphi(i, t) \geq 0, i \in \boldsymbol{K}_{t}, t \in \boldsymbol{T} .
\end{array}
$$


Inequality (25) comes from Equation (23). What we have to do next is to verify the duality between Problem $\left(C^{T}\right)$ and $\left(C^{S}\right)$. The verification would make sure that the minimax value of the expected payoff is equal to its maximin value and our game can be solved.

If we assign dual variables $\phi(i, t), y(i, t, s), z(i, t, s)$ and $\zeta(t)$ corresponding to condition $(17),(18),(19)$ and $(20)$, respectively, in Problem $\left(C^{T}\right)$, we can generate a dual problem as follows.

$$
\begin{aligned}
C D_{1}^{S} \quad: & \max \sum_{t \in \boldsymbol{T}} \zeta(t) \\
\text { s.t. } \quad & \sum_{t \in \boldsymbol{T}_{i \in \boldsymbol{K}_{t}}} \sum_{l} \boldsymbol{a}_{l}(i, t) \phi(i, t) \leq \Phi_{l}, l \in \boldsymbol{L} \\
& -\sum_{t \in T^{*}(\xi)} \sum_{i \in A^{*}(k, \xi)} c(i, k, \xi) \phi(i, t)-\left(1-\delta_{\xi T}\right) y(k, \xi, s)+\left(1-\delta_{\xi 1}\right) z(k, \xi, s)+\zeta(\xi)=0, \\
& y(k, \xi, s)-z(j, \xi+1, \Gamma(k, \xi, s, j)) \leq 0, \quad k \in \boldsymbol{K}_{\xi}, s \in \boldsymbol{S}_{\xi}, \xi \in \boldsymbol{T} \\
& \quad k \in \boldsymbol{K}_{\xi}, j \in B(k, \xi, s), s \in \boldsymbol{S}_{\xi}, \xi=1, \cdots, T-1
\end{aligned}
$$

In Equation (26), $\delta$. is the Kronecker's delta. Since $z(\cdot)$ has not been defined yet for index $\xi=1$, we use a definition $z(k, 1, s) \equiv 0$. From equation (26), we can replace variable $y(\cdot)$ with

$$
\begin{aligned}
y(k, \xi, s)=z(k, \xi, s)+\zeta(\xi)-\sum_{t \in T^{*}(\xi)} \sum_{i \in A^{*}(k, \xi)} c(i, k, \xi) \phi(i, t), \\
\quad k \in \boldsymbol{K}_{\xi}, s \in \boldsymbol{S}_{\xi}, \xi=1, \cdots, T-1
\end{aligned}
$$

and then delete variable $y(\cdot)$ from the formulation. Noting that Equation (26) gives $z(k, \xi, s)$ a constant value for $\xi=T$, we can make a new formulation as follows.

$$
\begin{array}{ll}
C D_{2}^{S} \quad: & \max \sum_{t \in \boldsymbol{T}} \zeta(t) \\
\text { s.t. } \quad & z(k, 1, s)=0, k \in \boldsymbol{K}_{1}, s \in \boldsymbol{S}_{1} \\
& z(k, \xi, s)+\zeta(\xi) \leq \sum_{t \in T^{*}(\xi)} \sum_{i \in A^{*}(k, \xi)} c(i, k, \xi) \phi(i, t)+z(j, \xi+1, \Gamma(k, \xi, s, j)), \\
& z(k, T, s)+\zeta(T)=\sum_{t \in T^{*}(T)} \sum_{i \in A^{*}(k, T)} c(i, k, T) \phi(i, t), k \in \boldsymbol{K}_{T}, s \in \boldsymbol{S}_{T} \\
& \sum_{t \in \boldsymbol{T}} \sum_{i \in \boldsymbol{K}_{t}} \boldsymbol{a}_{l}(i, t) \phi(i, t) \leq \Phi_{l}, l \in \boldsymbol{L} \\
& \phi(i, t) \geq 0, i \in \boldsymbol{K}_{t}, t \in \boldsymbol{T} .
\end{array}
$$

After a substitution of $w(k, \xi, s) \equiv z(k, \xi, s)+\sum_{t=\xi}^{T} \zeta(t)$ for $z(\cdot)$, a new formulation is 
generated.

$$
\begin{array}{ll}
C D_{3}^{S}: & \max \sum_{t \in \boldsymbol{T}} \zeta(t) \\
\text { s.t. } \quad w(k, 1, s) & =\sum_{t \in \boldsymbol{T}} \zeta(t), k \in \boldsymbol{K}_{1}, s \in \boldsymbol{S}_{1} \\
& w(k, \xi, s) \leq \sum_{t \in T^{*}(\xi)} \sum_{i \in A^{*}(k, \xi)} c(i, k, \xi) \phi(i, t)+w(j, \xi+1, \Gamma(k, \xi, s, j)), \\
& w(k, T, s)=\sum_{t \in T^{*}(T)} \sum_{i \in A^{*}(k, T)} c(i, k, T) \phi(i, t), k \in \boldsymbol{K}_{T}, s \in \boldsymbol{S}_{T} \\
& \sum_{t \in \boldsymbol{T}} \sum_{i \in \boldsymbol{K}_{t}} \boldsymbol{a}_{l}(i, t) \phi(i, t) \leq \Phi_{l}, l \in \boldsymbol{L}, \quad \phi(i, t) \geq 0, i \in \boldsymbol{K}_{t}, t \in \boldsymbol{T} .
\end{array}
$$

In this problem, the optimization process steps forward as follows. The last value of $w(k, \xi, s)$ for $\xi=T$ is given by Equation (30). Inequality (29) is regarded as a difference equation of $w(\cdot)$ between successive time points $\xi$ and $\xi+1$. While satisfying this inequality, the problem is going to increase an initial value $w(k, 1, s)$ for $\xi=1$ as large as possible by manipulating variable $\phi(\cdot)$. An optimal value is evidently nonnegative. Really, variables $\zeta(t)=0, \phi(i, t)=0, w(k, \xi, s)=0$ are feasible and they give an objective value zero. Judging from the optimization procedure, the value of the objective function is affected by $w(k, 1, s), k \in \boldsymbol{K}_{1}, s \in \boldsymbol{S}_{1}$. Problem $\left(C D_{3}^{S}\right)$ has a form of $\{\max \rho$ s.t. $w(k, 1, s)=\rho, k \in$ $\left.\boldsymbol{K}_{1}, s \in \boldsymbol{S}_{1}, \cdots\right\}$, but we can change it to $\left\{\max \rho\right.$ s.t. $\left.w(k, 1, s) \geq \rho, k \in \boldsymbol{K}_{1}, s \in \boldsymbol{S}_{1}, \cdots\right\}$ while keeping an optimal value unchanged by the following reason. Let $\rho^{*}$ be an optimal value for the latter problem. For $w(k, 1, s)>\rho^{*}, k \in \boldsymbol{K}_{1}, s \in \boldsymbol{S}_{1}$, we can change the variable to $w(k, 1, s)=\rho^{*}$ because changed variables are still feasible and the optimal value of the problem still remains same, as seen from the optimization structure of the problem. On the other hand, the latter problem has a larger optimal value than the former one. Now we have verified that both problems have the same optimal value and then Problem $\left(C D_{3}^{S}\right)$ is equivalent to $\left(C^{S}\right)$ and a dual variable $\phi(i, t)$ is nothing but a searcher's strategy $\varphi(i, t)$. At the same time, we have reached the proof of the duality between two problems $\left(C^{T}\right)$ and $\left(C^{S}\right)$.

\section{Numerical Examples}

Here we analyze two numerical examples. One is an example of Model A, stated in Section 2 , with additional assumptions $(\mathrm{C} 1) \sim(\mathrm{C} 4)$ in Section 4 . The other is taken to compare two different formulations $\left(G^{T}\right)$ and $\left(C^{T}\right)$.

In the first example, a searcher distributes durable and long-distance reachable resource to detect a target and the target expends its energy to move. A search space $\boldsymbol{K}$ consists of 37 equilateral hexagons, as Figure 1 shows, and a time space has 8 time points, $\boldsymbol{T}=\{1, \cdots, 8\}$. A target starts from cell 1 at time 1 and therefore, in Assumption (C1), a geographic space $\boldsymbol{K}_{t}$ is made as $\boldsymbol{K}_{1}=\{1\}$ for time 1 and $\boldsymbol{K}_{t}=\boldsymbol{K}$ for any other time $t$.

Cells $8,17,21,30,31$ are hazard cells and then the target cannot stay there. The hazard cell also interrupts the effect of searching resource. Reachable area $A(i)$ in Assumption (A3) is set such that the effect of searching resource reaches up to 2nd-neighbored cells from the point where the resources are allocated. "2nd-neighbored" means that a relevant 
cell is placed next to the neighborhood cell from the original point. For example, the resource in cell 7 has some effects on neighborhood cells 2,6,12,13,3 and 2nd-neighbored cells $1,5,11,18,19,20,14,4$, but no effect on cell 9 because the cell is just behind hazard cell 8. The attenuation rate $\beta(i, j)$ between cell $i$ and $j$, supposed in Assumption (A3), is an instance of parameter $c(i, j, t)$ in Assumption (C4). $\beta(i, j)$ is assumed to be 1 for just the same cell $j=i, 0.5$ for the neighborhood cell and 0.3 for the 2 nd-neighbored cell. But there are some exceptions related to the hazard cell. As stated before, if two cells $i$ and $j$ are placed just behind a hazard cell each other, searching resource in one cell has no effect on the other or $\beta(i, j)=0$. If a half part of one cell faces a half of the other cell having a hazard cell between them, the attenuation rate becomes less than usual or $\beta(i, j)=0.1$. For example, for cell $i=7$, neighborhood cell $j=2,6,12,13,3$ has rate $\beta(i, j)=0.5$ and 2nd-neighbored cell $j=1,5,11,18,19,20$ has $\beta(i, j)=0.3$, but cell $j=14,4$ has a smaller rate $\beta(i, j)=0.1$. We also assume that the effect of searching resource lasts during two time points or $t_{c}=1$ in Assumption (A3). We do not assume inequality (2) but assume (1) as the constraints on the distribution of searching resource. The searcher can use unit resource at most after time $\tau=2$, that is, $\Phi(1)=0, \Phi(2)=\cdots=\Phi(8)=1$.

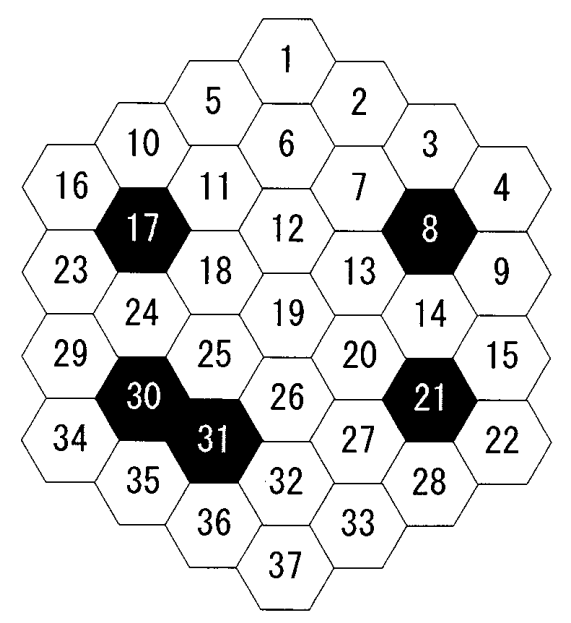

Figure 1: Search space

As an attribute of the target, mentioned in Assumption (C2), we think of energy. The target is able to move from his current cell to the region within the distance of 2ndneighbored cell. He can go through hazard cells $8,17,21,30,31$ but he cannot move into or stay in the cells. When the target moves to a neighborhood cell, his energy $e$ decreases by one and changes to $e-1$. After moving to a 2nd-neighbored cell, energy $e$ changes to $e-4$. The motion with the expenditure of more energy than his current energy is not permitted for the target. It does not require any energy to stay in the same cell so that the target is forced to continue staying after he exhausts his energy. The target has his initial energy 9 at time 1. Therefore the set of the target attribute $\boldsymbol{S}_{t}$, stated in Assumption (C2), is $\boldsymbol{S}_{1}=\{9\}$ for initial time $t=1$ and $\boldsymbol{S}_{t}=\{0, \cdots, 9\}$ for any other time $t$. The changing of the target energy explained above prescribes the transition of the target attribute $\Gamma(i, t, e, j)$.

Using the parameter setting above, we solve problem $\left(C^{T}\right)$ or $\left(C^{S}\right)$ to obtain optimal strategies of the searcher $\{\varphi(i, t), i \in \boldsymbol{K}, t \in \boldsymbol{T}\}$ and the target $\{q(i, t, e), i \in \boldsymbol{K}, t \in \boldsymbol{T}, e \in$ $\left.\boldsymbol{S}_{t}\right\}$. The optimal solution $\varphi(i, t)$ is shown in Table 2, where a row indicates a cell and a column a time point. For comprehensibility, we modify the optimal target strategy $q(i, t, e)$ 
by $q(i, t) \equiv \sum_{e \in \boldsymbol{S}_{t}} q(i, t, e)$, which means an optimal probability that the target is in cell $i$ at time $t$, and we illustrate the modified probabilities in Table 4.

Now let us transform the expression of the expected payoff (22) as follows.

$$
\begin{aligned}
\text { Expected payoff } & =\sum_{\xi \in \boldsymbol{T}} \sum_{k \in \boldsymbol{K}_{\xi}}\left(\sum_{t \in T^{*}(\xi)} \sum_{i \in A^{*}(k, \xi)} c(i, k, \xi) \varphi(i, t)\right) \sum_{s \in \boldsymbol{S}_{\xi}} q(k, \xi, s) \\
& =\sum_{\xi \in \boldsymbol{T}} \sum_{k \in \boldsymbol{K}_{\xi}}\left(\sum_{t \in T^{*}(\xi)} \sum_{i \in A^{*}(k, \xi)} c(i, k, \xi) \varphi(i, t)\right) q(k, \xi) .
\end{aligned}
$$

From the above, the accumulated amount of searching resources $\sum_{t \in T^{*}(\xi)} \sum_{i \in A^{*}(k, \xi)} c(i, k, \xi)$ $\varphi(i, t)$ is a weight on the target distribution $q(k, \xi)$ in cell $k$ at time $\xi$ and therefore the accumulated searching resource gives the target an important pointer about how he should shape his distribution in the search space. The accumulated resources are shown in Table 3, placed between Table 2 and 4 . The target has too complex constraints on his movement and his energy expenditure to generate a perfectly optimal formation of his existence probability $q(k, \xi)$ corresponding to the accumulated resources. We are interested in interpreting how well the target acts to equilibrate an optimal response to the accumulated resources and an optimal movement according to his constraints, as well as how optimal the searcher's strategy is.

Noting that the target starts from cell 1 with initial energy 9 , we can see that the target reaches every cell at time 6 at the earliest by jumping to a 2nd-neighbored cell once and walking to a neighborhood cell four times. Taking into account this information, we can itemize the following characteristics of the optimal strategy of the searcher in Table 2 .

(1) Table 2 has a sparse structure, where there are many zeros. Just after some resources are distributed once in a cell, there is no allocation of resource in the same cell. We can see such a periodical distribution of searching resource for many cells in Table 2 . Even the sparse structure of Table 2 generates the distribution of accumulated effective resources which widely covers the possible area of the target as time passes, as shown in Table 3.

(2) Cell 6 is a key position that the target passes through at the early time. Therefore the searcher should scatter a lot of resources there at time 2 but the importance of the cell 6 is lost after then.

(3) Cell 12 is a crucial position because the searching resource allocated there has a wide effect over the central area through the whole time.

(4) In the right half area of the search space, there are two hazard cells 8 and 21 . There are some resources distributed in cell 9 and 15, which cover the right area of the hazard cells. According to the expanding possible area of the target, the searcher regards cell 9 as an important cell to distribute resource at the early time near 3 and cell 15 as a key cell at the late time after 5 .

(5) Effects of resource in cell 12 cannot reach the lower right area of the search space around hazard cell 21 . Cell 22 and 27 becomes important after time 5 when the target possibly arrives there.

(6) To cover the upper left area of the search space around hazard cell 17, cell 16 is the most important, and two cells 10 and 23 play an auxiliary role. 
Table 2: Optimal distribution of searching resource

\begin{tabular}{|c|c|c|c|c|c|c|c|}
\hline cells \time & 2 & 3 & 4 & 5 & 6 & 7 & 8 \\
\hline 1 & 0 & 0 & 0.154 & 0 & 0.098 & 0 & 0.101 \\
\hline 2 & 0 & 0 & 0.026 & 0 & 0.042 & 0 & 0.017 \\
\hline 3 & 0.105 & 0 & 0 & 0.006 & 0.009 & 0 & 0.006 \\
\hline 4 & 0 & 0.210 & 0.047 & 0.139 & 0 & 0.161 & 0 \\
\hline 5 & 0 & 0 & 0.038 & 0 & 0.023 & 0.007 & 0.027 \\
\hline 6 & 0.262 & 0 & 0 & 0 & 0 & 0 & 0 \\
\hline 7 & 0 & 0 & 0 & 0 & 0 & 0 & 0 \\
\hline 8 & 0 & 0 & 0 & 0 & 0 & 0 & 0 \\
\hline 9 & 0 & 0.216 & 0.019 & 0.072 & 0 & 0.038 & 0 \\
\hline 10 & 0.105 & 0 & 0 & 0.015 & 0.006 & 0.003 & 0 \\
\hline 11 & 0 & 0 & 0 & 0 & 0 & 0 & 0 \\
\hline 12 & 0.529 & 0.146 & 0.405 & 0 & 0.320 & 0.040 & 0.320 \\
\hline 13 & 0 & 0 & 0 & 0 & 0 & 0 & 0 \\
\hline 14 & 0 & 0 & 0 & 0 & 0 & 0 & 0 \\
\hline 15 & 0 & 0 & 0 & 0.193 & 0 & 0.185 & 0 \\
\hline 16 & 0 & 0.222 & 0 & 0.164 & 0 & 0.145 & 0 \\
\hline 17 & 0 & 0 & 0 & 0 & 0 & 0 & 0 \\
\hline 18 & 0 & 0 & 0 & 0 & 0 & 0 & 0 \\
\hline 19 & 0 & 0 & 0 & 0 & 0 & 0 & 0 \\
\hline 20 & 0 & 0 & 0.065 & 0 & 0 & 0 & 0 \\
\hline 21 & 0 & 0 & 0 & 0 & 0 & 0 & 0 \\
\hline 22 & 0 & 0 & 0 & 0 & 0.112 & 0 & 0.088 \\
\hline 23 & 0 & 0.206 & 0.079 & 0 & 0.003 & 0.097 & 0 \\
\hline 24 & 0 & 0 & 0 & 0 & 0 & 0 & 0 \\
\hline 25 & 0 & 0 & 0.050 & 0 & 0 & 0 & 0 \\
\hline 26 & 0 & 0 & 0.117 & 0 & 0 & 0.002 & 0.041 \\
\hline 27 & 0 & 0 & 0 & 0.128 & 0.172 & 0.050 & 0.157 \\
\hline 28 & 0 & 0 & 0 & 0 & 0 & 0 & 0 \\
\hline 29 & 0 & 0 & 0 & 0.282 & 0 & 0.116 & 0 \\
\hline 30 & 0 & 0 & 0 & 0 & 0 & 0 & 0 \\
\hline 31 & 0 & 0 & 0 & 0 & 0 & 0 & 0 \\
\hline 32 & 0 & 0 & 0 & 0 & 0.181 & 0 & 0.155 \\
\hline 33 & 0 & 0 & 0 & 0 & 0 & 0 & 0 \\
\hline 34 & 0 & 0 & 0 & 0 & 0.033 & 0 & 0.059 \\
\hline 35 & 0 & 0 & 0 & 0 & 0 & 0.142 & 0.029 \\
\hline 36 & 0 & 0 & 0 & 0 & 0 & 0 & 0 \\
\hline 37 & 0 & 0 & 0 & 0 & 0 & 0.015 & 0 \\
\hline total & 1 & 1 & 1 & 1 & 1 & 1 & 1 \\
\hline
\end{tabular}


Table 3: Distribution of accumulated effective searching resources

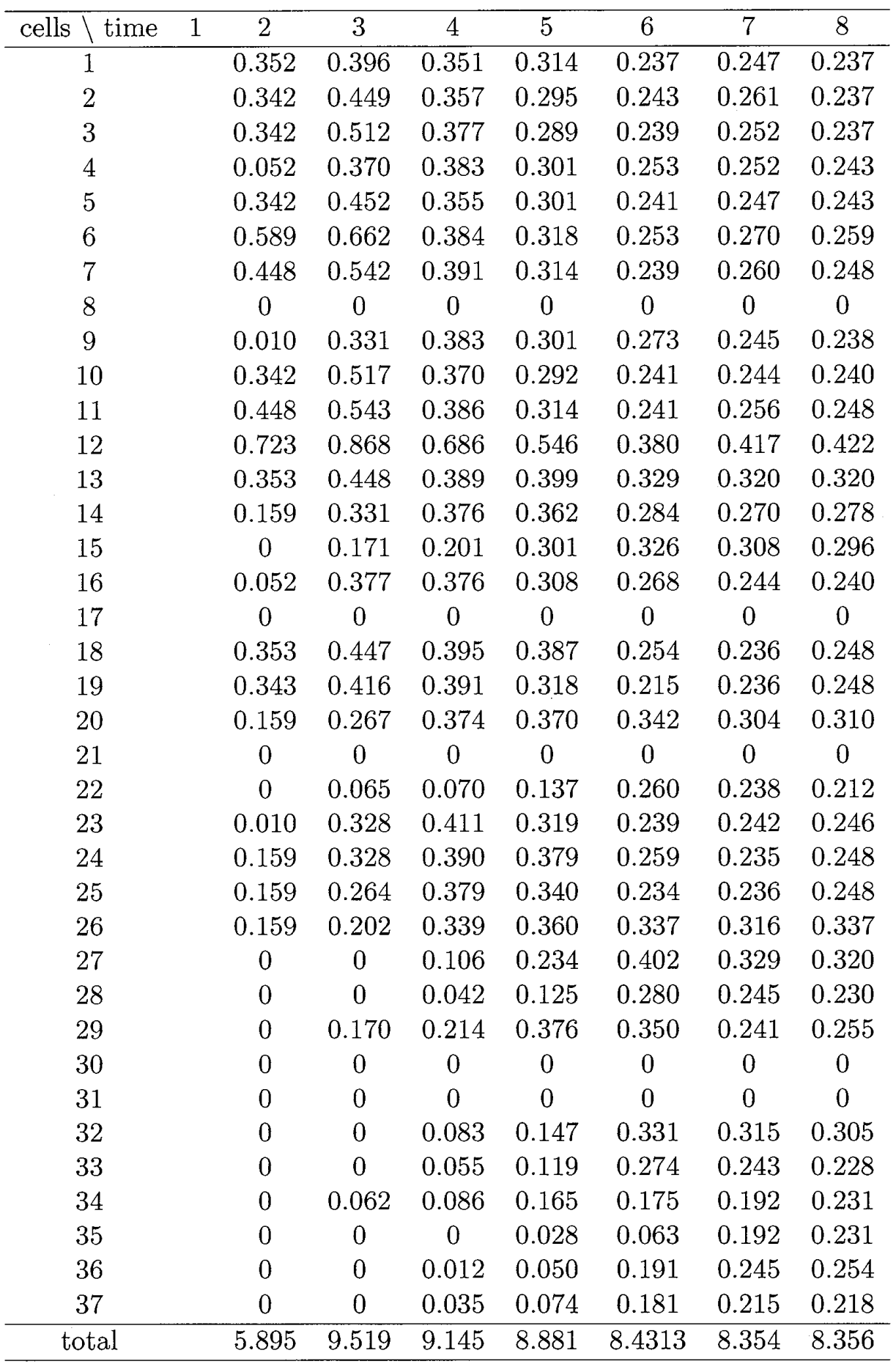


Table 4: Optimal distribution of target

\begin{tabular}{|c|c|c|c|c|c|c|c|c|}
\hline cells $\backslash$ time & 1 & 2 & 3 & 4 & 5 & 6 & 7 & 8 \\
\hline 1 & 1 & 0.005 & 0.153 & 0.102 & 0 & 0.045 & 0.046 & 0.047 \\
\hline 2 & 0 & 0.139 & 0 & 0.070 & 0.054 & 0.046 & 0.044 & 0.048 \\
\hline 3 & 0 & 0.262 & 0 & 0.030 & 0.101 & 0.067 & 0.063 & 0.061 \\
\hline 4 & 0 & 0 & 0.162 & 0.075 & 0.011 & 0.040 & 0.039 & 0.039 \\
\hline 5 & 0 & 0.145 & 0 & 0.057 & 0.033 & 0.039 & 0.033 & 0.033 \\
\hline 6 & 0 & 0.055 & 0 & 0 & 0.031 & 0 & 0 & 0 \\
\hline 7 & 0 & 0.078 & 0 & 0 & 0 & 0 & 0 & 0 \\
\hline 8 & 0 & 0 & 0 & 0 & 0 & 0 & 0 & 0 \\
\hline 9 & 0 & 0 & 0.070 & 0.053 & 0.089 & 0.046 & 0.051 & 0.050 \\
\hline 10 & 0 & 0.282 & 0 & 0.054 & 0.094 & 0.057 & 0.060 & 0.060 \\
\hline 11 & 0 & 0 & 0.032 & 0 & 0.010 & 0.020 & 0 & 0.001 \\
\hline 12 & 0 & 0.034 & 0 & 0 & 0 & 0 & 0 & 0 \\
\hline 13 & 0 & 0 & 0.022 & 0 & 0 & 0 & 0 & 0 \\
\hline 14 & 0 & 0 & 0.062 & 0.046 & 0 & 0 & 0 & 0 \\
\hline 15 & 0 & 0 & 0 & 0.047 & 0.111 & 0.047 & 0.047 & 0.047 \\
\hline 16 & 0 & 0 & 0.167 & 0.048 & 0.048 & 0.048 & 0.054 & 0.055 \\
\hline 17 & 0 & 0 & 0 & 0 & 0 & 0 & 0 & 0 \\
\hline 18 & 0 & 0 & 0 & 0.032 & 0 & 0 & 0.028 & 0.021 \\
\hline 19 & 0 & 0 & 0.055 & 0 & 0.016 & 0.057 & 0.040 & 0.042 \\
\hline 20 & 0 & 0 & 0.078 & 0.037 & 0 & 0 & 0 & 0 \\
\hline 21 & 0 & 0 & 0 & 0 & 0 & 0 & 0 & 0 \\
\hline 22 & 0 & 0 & 0 & 0 & 0 & 0.078 & 0.078 & 0.079 \\
\hline 23 & 0 & 0 & 0.060 & 0.081 & 0.031 & 0.039 & 0.034 & 0.031 \\
\hline 24 & 0 & 0 & 0.105 & 0.031 & 0 & 0 & 0 & 0.002 \\
\hline 25 & 0 & 0 & 0 & 0.046 & 0.078 & 0.046 & 0.053 & 0.054 \\
\hline 26 & 0 & 0 & 0.034 & 0.055 & 0 & 0 & 0 & 0 \\
\hline 27 & 0 & 0 & 0 & 0.062 & 0.084 & 0.062 & 0.062 & 0.062 \\
\hline 28 & 0 & 0 & 0 & 0 & 0 & 0 & 0 & 0 \\
\hline 29 & 0 & 0 & 0 & 0.039 & 0.120 & 0.039 & 0.044 & 0.039 \\
\hline 30 & 0 & 0 & 0 & 0 & 0 & 0 & 0 & 0 \\
\hline 31 & 0 & 0 & 0 & 0 & 0 & 0 & 0 & 0 \\
\hline 32 & 0 & 0 & 0 & 0.034 & 0.089 & 0.034 & 0.034 & 0.034 \\
\hline 33 & 0 & 0 & 0 & 0 & 0 & 0.022 & 0 & 0 \\
\hline 34 & 0 & 0 & 0 & 0 & 0 & 0.081 & 0.054 & 0.062 \\
\hline 35 & 0 & 0 & 0 & 0 & 0 & 0.032 & 0.068 & 0.065 \\
\hline 36 & 0 & 0 & 0 & 0 & 0 & 0.009 & 0 & 0 \\
\hline 37 & 0 & 0 & 0 & 0 & 0 & 0.046 & 0.068 & 0.068 \\
\hline total & 1 & 1 & 1 & 1 & 1 & 1 & 1 & 1 \\
\hline
\end{tabular}


(7) The lower left area is difficult for the searcher to cover by his searching resource because there are two hazard cells 30 and 31 . The searcher uses three cells $32,34,35$ for the distribution of resource after time 6 when the target can arrive the area.

The above way that the searcher distributes his searching resource generates the map of accumulated searching resources, as seen in Table 3. We pick up some characteristics from the table.

(1) Because the searcher constantly allocates his searching resource to cell 12, a lot of accumulated resources are stacked there and they bear the accumulation of effective resources in neighborhood cells 7 and 11 .

(2) At the early time 2, more effective resources are accumulated in the upper area near a starting point of the target, but at time $3 \sim 5$, the amount of the accumulated resources is getting larger in cell 9,14,16,23,24 around hazard cell 8 and 17. In other cells, we can see that some resources are accumulated, which is caused by the characteristic distribution of resource featured by Item (4) and (6) described above for Table 2, although the target has not arrived there yet, as seen from Table 4.

(3) After the target reaches all the search space at time 6, the accumulated resources spread evenly all over the space. The distribution of the accumulated resources is getting much even because the minimum amount of accumulated resources becomes larger such as $0.063,0.192$ and 0.212 at time 6,7 and 8 , respectively.

The following characteristics appear in Table 4, as concerns an optimal distribution of the target.

(1) After the target can reach all over the search space at time 6, the distribution of the target spreads widely and the probability of the target existence is less than 0.08 everywhere. However the distribution cannot be perfectly uniform because the target that arrives at the bottom area does not have energy enough to move further and rearrange his distribution more uniformly.

(2) Cell 12 is a key position that the target goes through at the early time to attain a wide diffusion over the search space. That is why there are a little probabilities of target existence in the cell at time 2. At other time, the target always avoids the cell because a lot of searching resources are always effective there. For cell 6 , we can find similar characteristics at the early time. In these cells 6,12 and their neighborhood cells $7,13,18$, there is almost no probability of target existence because the resource allocated there has wide effects all over the upper central area without any interruption by the hazard cells.

(3) After the searcher begins to distribute searching resource in cell 15,22 and 27 at time 5 , their neighbor cells $28,14,20,26$ are assigned no probability of the target existence because effects of the resource distributed in cell 6 and 12 are overlapped.

(4) As mentioned in characteristic (6) and (7) about Table 2, in the left half area of the search space, the searcher distributes some searching resources in cell 10,16,23 at the early time and in $32,34,35$ at the late time. In their neighbor cells 24,36 , additional effects of the resource allocated to cell 12,27 make the probability of the target existence zero.

(5) In cells $15,22,27,10,16,23,32,34$ and 35 that we mentioned in Item (3) and (4) above, there are a little probabilities of the target. Wide effects of resources distributed in those cells are interrupted by hazard cells and cannot be accumulated there enough to make the probability of target existence zero. Additionally, if the target avoids those cells completely, the probability of target existence is biased in smaller area, which would 
help the searcher do more effective search.

We have itemized some characteristics about the optimal strategies of players. As a result, we can see that attributes of searching resource, such as durability and long-distance reachability, and the constraints on the target motion complexly affect the optimal strategies of players in the characteristic search space with some hazard cells.

We propose two types of formulations in Section 3 and 4. The most general formulation is $\left(G^{T}\right)$ corresponding to Model G. From applying the concrete parameter setting to $\left(G^{T}\right)$, we generate other formulations $\left(P_{B}^{T}\right)$ and $\left(P_{A}^{T}\right)$, which correspond to Model Basic and Model A, respectively. That is why there is no essential difference between those three formulations $\left(G^{T}\right),\left(P_{B}^{T}\right)$ and $\left(P_{A}^{T}\right)$. As stated at the beginning of Section 4 , we devise another formulation $\left(C^{T}\right)$ by using target distribution $q(i, t, s)$ and transition probability $v(i, j, t, s)$ to break the shortcoming of the formulation $\left(G^{T}\right)$ in terms of the sensitivity for the size of time space. To figure out the efficiency of $\left(C^{T}\right)$, now let us compare two formulations $\left(G^{T}\right)$ and $\left(C^{T}\right)$ by a numerical example, which is carried out by varying the upper limit of time $T$ in the example of Figure 1 while keeping any other parameter unchanged such as $\tau=2, t_{c}=$ $1, \Phi(t)=1, t \in \widehat{T}$ and so on.

The example by Figure 1 is made by adding energy constraints on target motion to Model A. Then we have two methods by formulation $\left(P_{A}^{T}\right)$ and $\left(C^{T}\right)$ to solve the problem. For the formulation $\left(P_{A}^{T}\right)$, we have to prepare an input data file of parameters $\Omega$ and $\Omega_{j \xi}$, which we make after enumerating all target paths in Figure 1 by specifying possible cells of the target at every time $t=1, \cdots, T$ under energy constraints. The number of all target paths or the cardinality of $\Omega$ would respond sensitively to the increase of $T$ to become larger, as we point out at the beginning of Section 4 . In formulation $\left(P_{A}^{T}\right)$, we use the probability of selecting paths, $\{\pi(\omega), \omega \in \Omega\}$, as variable and then the number of the variables is almost the same as the cardinality $|\Omega|$. Considering that the constraints of $\left(P_{A}^{T}\right)$ are simpler than $\left(C^{T}\right)$, we could easily solve the problem with small $\Omega$ by $\left(P_{A}^{T}\right)$ in the case of small $T$ but not easily in the case of large $T$. On the other hand, formulation $\left(C^{T}\right)$ has variables with an index $s$ indicating energy state beside indices of cell $i$ and time $t$. The number of variables increases as $T$ becomes larger but we can imagine that the increasing ratio would be linearly unlike the variables of $\left(P_{A}^{T}\right)$.

Table 5 shows the value of the game, abbreviated to 'val.', the number of variables ("\# of var.'), the number of constraints ('\# of func.'), computational time ('CPU(second)'), the size of prepared input data file ('IDF (Kilobyte)') and the cardinality $|\Omega|$ when we solve each problem with varying $T=3, \cdots, 10$ by two formulations. Figures in the upper position are for $\left(P_{A}^{T}\right)$ and those in the lower position are for $\left(C^{T}\right)$. We use a personal computer SOTEC G4170R with Pentium 4 of $1.7 \mathrm{GHz}$ and a mathematical programming software NUOPT Ver.5.0 (Mathematical Systems Inc.) for the computation. For equal to or larger than $T=6$, the number of target paths becomes too large for NUOPT to expand the formulation $\left(P_{A}^{T}\right)$ in the concrete, as seen in the column of ' $\operatorname{IDF}(\mathrm{Kb})$ ' or ' $|\Omega|$. That is why there is no data for $\left(P_{A}^{T}\right)$ in the case of $T=6 \sim 10$. We can see that the computational time of $\left(P_{A}^{T}\right)$ increases in an exponential ratio for $T=3,4$ and 5 even though $\left(P_{A}^{T}\right)$ has smaller computational time for those $T \mathrm{~s}$. On the other hand, the numbers of variables and constraints of $\left(C^{T}\right)$ increase approximately linearly to $T$ and its computational time becomes larger in a little stronger ratio than linear one. By the sensitivity analysis on $T$, we can verify that formulation $\left(C^{T}\right)$ is superior to $\left(P_{A}^{T}\right)$ for the problem with large time space. However, we might note that $\left(P_{A}^{T}\right)$ takes a very little time to solve the problem with the small size of path set, as in the case of $T=3,4$. 
Table 5: Comparison of two formulations

\begin{tabular}{|c|c|c|c|c|c|c|}
\hline$T$ & val. & \# of var. & \# of func. & $\mathrm{CPU}(\mathrm{sec})$ & IDF $(\mathrm{Kb})$ & $|\Omega|$ \\
\hline \multirow[t]{2}{*}{3} & 0.72 & 110 & 76 & 0.7 & 16 & 108 \\
\hline & & 42183 & 1558 & 19.0 & 32 & \\
\hline \multirow[t]{2}{*}{4} & 1.00 & 1070 & 113 & 2.7 & 32 & 1067 \\
\hline & & 56244 & 2336 & 58.4 & 32 & \\
\hline \multirow[t]{2}{*}{5} & 1.31 & 5535 & 150 & 30.5 & 146 & 5531 \\
\hline & & 70305 & 3114 & 85.6 & 32 & \\
\hline \multirow[t]{2}{*}{6} & & - & - & - & 956 & 28593 \\
\hline & 1.60 & 84366 & 3892 & 150.2 & 32 & \\
\hline \multirow[t]{2}{*}{7} & & - & - & - & - & 166232 \\
\hline & 1.86 & 98427 & 4670 & 235.0 & 32 & \\
\hline \multirow[t]{2}{*}{8} & & - & - & - & - & 749799 \\
\hline & 2.11 & 112488 & 5448 & 290.9 & 32 & \\
\hline \multirow[t]{2}{*}{9} & & - & - & - & - & 2746986 \\
\hline & 2.35 & 126549 & 6226 & 362.0 & 32 & \\
\hline \multirow[t]{2}{*}{10} & & - & - & - & - & 9323260 \\
\hline & 2.60 & 140610 & 7004 & 425.1 & 32 & \\
\hline
\end{tabular}

\section{Conclusion}

This paper deals with a two-person zero-sum game called search allocation game (SAG), where a searcher distributes his searching resource to detect a target and the target moves around to evade the searcher. Practical searching resource has a variety of properties on its effects and constraints. We take several examples of search games, called Model A and Model Basic, to illustrate properties of searching resource and distill linear expressions of resource for the payoff and the constraints. There have been few researches on the SAG with complex attributes of searching resource. By this reason, we formulate a general model of the SAG with a linear payoff and linear constraints of searching resource. In result, we verify that there exists an equilibrium with a pure strategy of the searcher and a mixed strategy of the target and we give a pair of linear programming formulations, which are dual to each other, for the solution of the game. The proposed method has flexibility and generality to be applied to many specialized instances.

We additionally developed another linear programming formulation to handle a large size of the problem in practical computational time by embedding specific conditions of the target movement. For the additional formulation, we devise a recursive equation by a dynamic programming. I could say again that proposed methods are useful for many applications to practical problems.

\section{References}

[1] S.S. Brown: Optimal search for a moving target in discrete time and space. Operations Research, 28 (1980), 1275-1286.

[2] F. Dambreville and J.P. Le Cadre: Detection of a Markovian target with optimization of the search efforts under generalized linear constraints. Naval Research Logistics, 49 (2002), 117-142.

[3] F. Dambreville and J.-P. Le Cadre: Search game for a moving target with dynamically generated informations. Proceedings of the 5th International Conference on Information 
Fusion (FUSION'2002) (2002), 243-250.

[4] J.M. Danskin: A helicopter versus submarine search game. Operations Research, 16 (1968), 509-517.

[5] J.M. Dobbie: A two-cell model of search for a moving target. Operations Research, 22 (1974), 79-92.

[6] J.N. Eagle and A.R. Washburn: Cumulative search-evasion games. Naval Research Logistics, 38 (1991), 495-510.

[7] A.Y. Garnaev: Search Games and Other Applications of Game Theory (SpringerVerlag, Tokyo, 2000).

[8] J. de Guenin: Optimum distribution of effort: an extension of the Koopman basic theory. Operations Research, 9 (1961), 1-9.

[9] O. Hellman: On the optimal search for a randomly moving target. SIAM Journal on Applied Mathematics, 22 (1972), 545-552.

[10] R. Hohzaki: Search allocation game. European Journal of Operational Research, 172 (2006), 101-119.

[11] R. Hohzaki: A search game taking account of attributes of searching resources. Naval Research Logistics, 55 (2008), 76-90.

[12] R. Hohzaki and K. Iida: A search game with reward criterion. Journal of the Operations Research Society of Japan, 41 (1998), 629-642.

[13] R. Hohzaki and K. Iida: A solution for a two-person zero-sum game with a concave payoff function. In W. Takahashi and T. Tanaka (eds.): Nonlinear Analysis and Convex Analysis (World Science Publishing Co., London, 1999), pp.157-166.

[14] R. Hohzaki, K. Iida, and T. Komiya: Discrete search allocation game with energy constraints. Journal of the Operations Research Society of Japan, 45 (2002), 93-108.

[15] R. Hohzaki and A. Washburn: An approximation for a continuous datum search game with energy constraint. Journal of the Operations Research Society of Japan, 46 (2003), 306-318.

[16] T. Ibaraki and N. Katoh: Resource Allocation Problems: Algorithmic Approaches (The MIT Press, London, 1988).

[17] K. Iida: An optimal distribution of searching effort for a moving target (in Japanese). Keiei Kagaku, 16 (1972), 204-215.

[18] K. Iida, R. Hohzaki, and S. Furui: A search game for a mobile target with the conditionally deterministic motion defined by paths. Journal of the Operations Research Society of Japan, 39 (1996), 501-511.

[19] K. Iida, R. Hohzaki, and K. Sato: Hide-and-search game with the risk criterion. Journal of the Operations Research Society of Japan, 37 (1994), 287-296.

[20] J.B. Kadane: Discrete search and the Neyman-Pearson lemma. Journal of Mathematical Analysis and Applications, 22 (1968), 156-171.

[21] Y.C. Kan: Optimal search of a moving target. Operations Research, 25 (1977), 864-870.

[22] K. Kikuta: A search game with traveling cost. Journal of the Operations Research Society of Japan, 34 (1991), 365-382.

[23] B.O. Koopman: The theory of search III: the optimum distribution of searching effort. Operations Research, 5 (1957), 613-626.

[24] T. Nakai: A sequential evasion-search game with a goal. Journal of the Operations Research Society of Japan, 29 (1986), 113-122. 
[25] T. Nakai: Search models with continuous effort under various criteria. Journal of the Operations Research Society of Japan, 31 (1988), 335-351.

[26] S.M. Pollock: A simple model of search for a moving target. Operations Research, 18 (1970), 883-903.

[27] L.D. Stone: Theory of Optimal Search (Academic Press, New York, 1975).

[28] W.R. Stromquist and L.D. Stone: Constrained optimization of functionals with search theory applications. Mathematics of Operations Research, 16 (1981), 518-529.

[29] A.R. Washburn: Search-evasion game in a fixed region. Operations Research, 28 (1980), 1290-1298.

[30] A.R. Washburn: Search for a moving target: the FAB algorithm. Operations Research, 31 (1983), 739-751.

Ryusuke Hohzaki

Department of Computer Science

National Defense Academy

1-10-20 Hashirimizu, Yokosuka,

239-8686, Japan

E-mail: hozaki@cc.nda.ac.jp 